Article

\title{
An Optical Sensor for Measuring the Position and Slanting Direction of Flat Surfaces
}

\author{
Yu-Ta Chen ${ }^{1}$, Yen-Sheng Huang ${ }^{1}$ and Chien-Sheng Liu ${ }^{1,2, *}$ \\ 1 Department of Mechanical Engineering and Advanced Institute of Manufacturing with High-Tech Innovations, \\ National Chung Cheng University, Chiayi County 62102, Taiwan; d04420006@ccu.edu.tw (Y.-T.C.); \\ g01420122@ccu.edu.tw (Y.-S.H.) \\ 2 Graduate Institute of Opto-Mechatronics, National Chung Cheng University, Chiayi County 62102, Taiwan \\ * Correspondence: imecsl@ccu.edu.tw; Tel.: +886-5-272-9306; Fax: +886-5-272-0589
}

Academic Editor: Changzhi Li

Received: 23 May 2016; Accepted: 5 July 2016; Published: 9 July 2016

\begin{abstract}
Automated optical inspection is a very important technique. For this reason, this study proposes an optical non-contact slanting surface measuring system. The essential features of the measurement system are obtained through simulations using the optical design software Zemax. The actual propagation of laser beams within the measurement system is traced by using a homogeneous transformation matrix (HTM), the skew-ray tracing method, and a first-order Taylor series expansion. Additionally, a complete mathematical model that describes the variations in light spots on photoelectric sensors and the corresponding changes in the sample orientation and distance was established. Finally, a laboratory prototype system was constructed on an optical bench to verify experimentally the proposed system. This measurement system can simultaneously detect the slanting angles $(x, z)$ in the $x$ and $z$ directions of the sample and the distance $(y)$ between the biconvex lens and the flat sample surface.
\end{abstract}

Keywords: flat surfaces; autofocus; autocollimator

\section{Introduction}

Among the measurement methods used in present day production lines, the non-contacting measurement technique is widely applied [1,2]. This technique is mainly divided into two types: image-based measurement and optical measurement. Image-based measurement focuses on image processing of captured images. The biggest limitations of this method are its long system response time and low accuracy. For these reasons, the technique has been gradually replaced in recent years by optical measurement. In the field of optical measurements, the positioning technique is of central importance [3,4]. For the laser positioning measurement technique, our research team has proposed a series of innovative system frameworks and combined them with optical diffusers to suppress the geometrical fluctuations of the laser beam and to achieve long-range and high-accuracy measurements [5-7]. Taken together, it is clear that optical measurement systems have the advantages of high measurement speed and accuracy.

In recent years, the positioning and slanting of a plane specular sample to be measured or processed has an important requirement in many fields of research and industry [8]. In applications of flat surface measurement techniques, Lee and Chang developed a laser scanning sensor with multiple position-sensitive device (PSD) detectors that can digitize a freeform surface through mathematical calculations using the Lambert model and geometric triangulation measurements [9]. The unique characteristic of this system is the use of multiple PSD detectors to resolve the dead zone of the measured angle and to broaden its range. Shiou developed a detector for distance and slanting angle measurements that can obtain the flat surface distance and slanting angle of the sample after the 
data are processed by triangulation operations [10]. Hirata and Haraguchi irradiated a sample with an annular laser beam and found that the shape of the laser beam changes with different slanting angles of the sample; by analyzing these changes, information on the slanting angles of the sample could be obtained. Finally, Gao developed a two-dimensional angle probe to measure the flatness of large silicon wafers [11]. Moreover, many methods are available to measure the parameters of slanting flat surfaces. However, to the best of our knowledge, these systems have complicated structures and high manufacturing costs. At present, there is no available measurement system with a simple architecture and straightforward assembly that can simultaneously measure the tilt angle and position information of a flat surface. Therefore, the aim of this study is to propose a fire-new measurement system with a simple architecture, straightforward assembly, and lower manufacturing cost that can simultaneously detect the tilt angle and position information of the sample.

\section{Architecture and Measurement Method of Proposed Measurement System}

The architecture of the proposed measurement system in this study consists of a He-Ne laser as the light source, two beam splitters (BS) accompanied by two biconvex lenses, and two charge-coupled devices (CCD) that are used as the photoelectric sensors to receive the optical signals. As shown in Figure 1, this design is an adaptation of an auto-focusing technique, namely, the triangulation method. The laser beam is directed off the optical axis by a small distance (s) and is then reflected by $\mathrm{BS}_{2}$ to become incident on the sample through the biconvex lens. The laser beam is then reflected by the sample to pass through the biconvex lens back to $\mathrm{BS}_{2}$ where the beam was split into two beams. One of the split beams goes directly through $\mathrm{BS}_{2}$ and is incident on $\mathrm{CCD}_{2}$. Notably, the laser spots on $\mathrm{CCD}_{2}$ will change as a function of the different sample orientations and distance. The other beam is reflected by beam splitter $\mathrm{BS}_{1}$ and travels through the biconvex lens before proceeding to $C C D_{1}$. The information of the laser spots on $\mathrm{CCD}_{1}$ will depend not only on the sample surface orientation but also on the change in the distance between the biconvex lens and the sample.

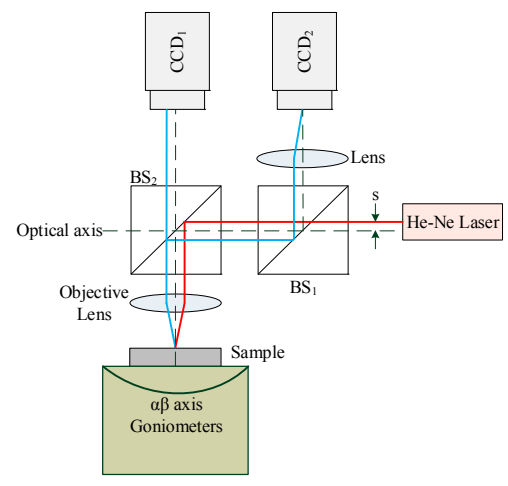

Figure 1. Architecture of proposed measurement system.

When using this proposed measurement system to measure the distance $\left(\delta_{y}\right)$ between the biconvex lens the flat sample surface and the slanting angles in the $x$ and $z$ directions $\left(\omega_{x}, \omega_{z}\right)$, the shape and center position of the laser beam received by the two CCDs will change as a function of both changes in the distance $\left(\delta_{y}\right)$ and slanting angles $\left(\omega_{x}, \omega_{z}\right)$. In addition, the received photoelectric signal contains factors that are influenced by the slanting angles $\left(\omega_{x}, \omega_{z}\right)$ of the sample when the sample distance $\left(\delta_{y}\right)$ resolved by the two CCDs is measured. Therefore, light beam tracing equations must be established via a HTM and skew-ray tracing to discuss the relationship between the sample distance $\left(\delta_{y}\right)$, slanting angles $\left(\omega_{x}, \omega_{z}\right)$, and the corresponding change in the center position of the laser spot. In this regard, a detailed mathematical derivation will be presented in Section 4. 


\section{Optical Simulation of Proposed Measurement System}

The ray trace function of the commercially available optical simulation software Zemax (Radiant Zemax LLC, Redmond, WA, USA) was used to simulate the actual ray propagation in the proposed measurement system. The Matlab software (The MathWorks, Inc., Natick, MA, USA) was then used to process the images of the laser spot position map, to calculate the location of the spot center, and to plot graphs for the spot position versus sample surface orientation and distance. In doing so, the feasibility of the proposed measurement system is determined and the measurement trend is determined.

Figure 2 shows the changes in the laser spots on $C C D_{1}$ and $C C D_{2}$ when the sample distance $(\delta y)$ is within the range of $-1 \mathrm{~mm} \sim+1 \mathrm{~mm}$ and the slanting angles $\left(\omega_{x}, \omega_{z}\right)$ are within the range of $-2^{\circ} \sim+2^{\circ}$. The figure indicates that the laser spots on $C C D_{1}$ and $C C_{2}$ show a changing trend when the sample experiences a small change in angle and distance. However, the relationship is not linear, and thus, the sample distance and orientation cannot be determined directly from the simulation results. Therefore, it is necessary to derive a mathematical measurement equation to analyze the relation between the sample distance/orientation and the two CCDs to obtain a quantitative mathematical analysis of the proposed measurement system.

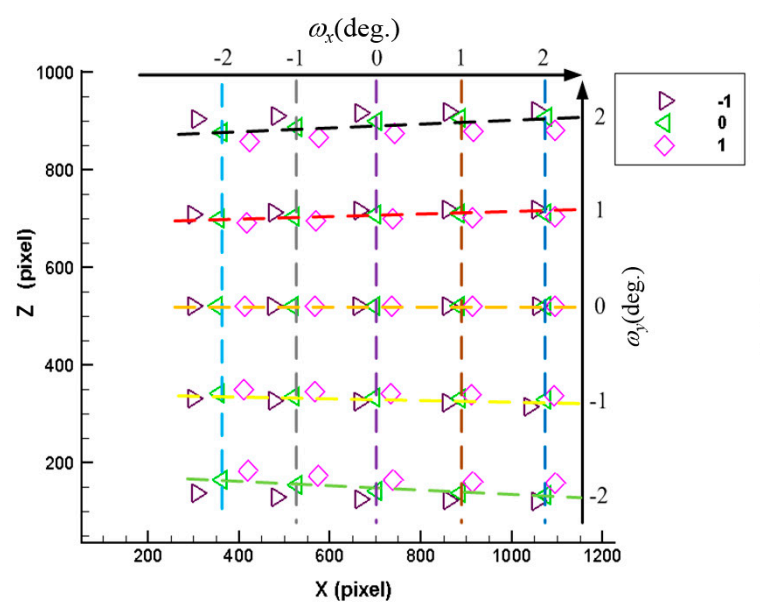

(a)

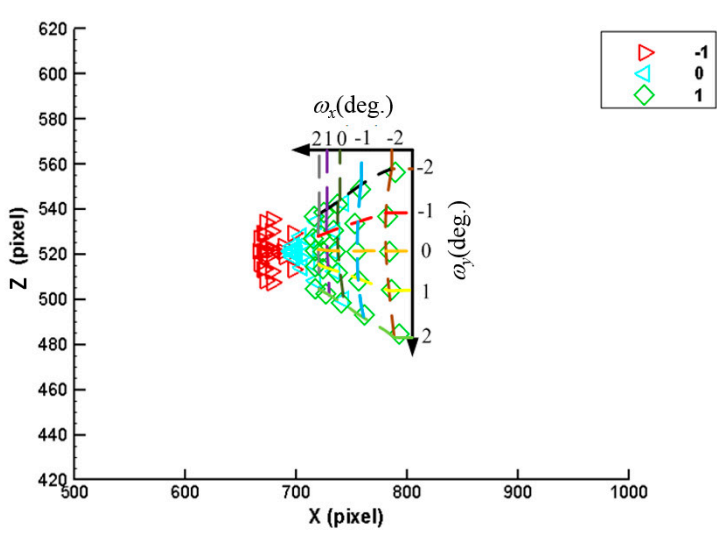

(b)

Figure 2. Optical simulation results: (a) changes in laser spots on $\mathrm{CCD}_{1}$ and (b) changes in laser spots on $\mathrm{CCD}_{2}$.

\section{Mathematical Modeling of Proposed Measurement System and Derivation of Actual Ray Path}

Mathematical modeling is established by using a HTM and the skew-ray tracing method proposed by Lin to model and perform ray tracing for the proposed measurement system architecture in this paper $[12,13]$. The HTM corresponding to the coordinate frame of each optical boundary relative to a reference coordinate system $(x y z)_{0}$ is defined sequentially. A mathematical model is proposed to develop a systematic forward and reverse mathematical derivation. Then, a linear equation of the measurement system is obtained by using a first-order Taylor series expansion to evaluate the first-order optical properties of the proposed measurement system.

\subsection{Establishing Optical Boundaries for Proposed Measurement System}

Based on the modeling steps in the skew-ray tracing method proposed by Lin $[12,13]$, the optical boundaries of the system ${ }^{i} \overline{r_{i}}$ are first defined. The proposed measurement system contains two parts: ray path I, which contains a total of 14 optical boundaries, and ray path II, which contains a total of eight optical boundaries, as shown in Figure 3: 


$$
{ }^{0} \overline{A_{i}}=\left[\begin{array}{cccc}
I_{i x} & J_{i x} & K_{i x} & t_{i x} \\
I_{i y} & J_{i y} & K_{i y} & t_{i y} \\
I_{i z} & J_{i z} & K_{i z} & t_{i z} \\
0 & 0 & 0 & 1
\end{array}\right]
$$

The transformation matrices of coordinate frames $(x y z)_{i}$ of each optical boundary ${ }^{i} \overline{r_{i}}$ relative to the reference coordinate frame $(x y z)_{0}$ are established as Equation (1) using the HTM. The elements in the HTM for each optical boundary are shown in Tables 1 and 2. The notations $C$ and $S$ denote cosine and sine, respectively. As seen, given are the HTM tables of coordinate $(x y z)_{i}$ for each optical boundary $i \overline{r_{i}}$ relative to the reference coordinate system of ray paths I and II, respectively, where $\omega_{x}, \omega_{z}$, and $\delta_{y}$ represent the rotation angle in the $x$ and $z$ directions and the distance $\left(\delta_{y}\right)$ between the biconvex lens and the flat sample surface. The initial position of the laser light source is defined as $P_{0}=\left[\begin{array}{ll}0 & 0-21\end{array}\right]^{\mathrm{T}}$, and its unit vector is defined as $\ell_{0}=\left[\begin{array}{llll}0 & 1 & 0 & 0\end{array}\right]^{\mathrm{T}}$.

Table 1. Parameters of the optical boundary coordinate transformation matrix for ray path I.

\begin{tabular}{cccccccc}
\hline $\mathbf{I}$ & $\mathbf{I}=\mathbf{1}$ & $\mathbf{I}=\mathbf{2}$ & $\mathbf{I}=\mathbf{3}$ & $\mathbf{I}=\mathbf{4}$ & $\mathbf{I}=\mathbf{5}$ & $\mathbf{I}=\mathbf{6}$ & $\mathbf{I}=\mathbf{7}$ \\
\hline \multirow{2}{*}{ Type } & $\begin{array}{c}\text { Convex } \\
\text { Spherical }\end{array}$ & $\begin{array}{c}\text { Concave } \\
\text { Spherical }\end{array}$ & $\begin{array}{c}\text { Flat Mirror } \\
\text { (Sample) }\end{array}$ & $\begin{array}{c}\text { Convex } \\
\text { Spherical }\end{array}$ & $\begin{array}{c}\text { Concave } \\
\text { Spherical }\end{array}$ & $\begin{array}{c}\text { Flat } \\
\text { Refracting }\end{array}$ & $\begin{array}{c}\text { Flat } \\
\text { Mirror }\end{array}$ \\
\hline $\mathrm{N}_{\mathrm{i}}$ & $1 / 1.515$ & 1.515 & Reflected & $1 / 1.515$ & 1.515 & $1 / 1.515$ & \\
$\mathrm{R}_{\mathrm{i}}$ & 24.397 & 24.397 & & 24.397 & 24.397 & & \\
$I_{i x}$ & 1 & 1 & 1 & 1 & 1 & 1 & -1 \\
$I_{i y}$ & 0 & 0 & $\mathrm{~S} \omega_{z}$ & 0 & 0 & 0 & 0 \\
$I_{i z}$ & 0 & 0 & 0 & 0 & 0 & 0 & 0 \\
$J_{i x}$ & 0 & 0 & $-\mathrm{C} \omega_{x} \mathrm{~S} \omega_{z}$ & 0 & 0 & 0 & 0 \\
$J_{i y}$ & 1 & 1 & 1 & 1 & 1 & 1 & $-\sqrt{2} / 2$ \\
$J_{i z}$ & 0 & 0 & $\mathrm{C} \omega_{x} \mathrm{~S} \omega_{z}$ & 0 & 0 & 0 & $\sqrt{2} / 2$ \\
$K_{i x}$ & 0 & 0 & $\mathrm{~S} \omega_{x} \mathrm{~S} \omega_{z}$ & 0 & 0 & 0 & 0 \\
$K_{i y}$ & 0 & 0 & $\mathrm{~S} \omega_{x} \mathrm{C} \omega_{z}$ & 0 & 0 & 0 & $\sqrt{2} / 2$ \\
$K_{i z}$ & 1 & 1 & 1 & 1 & 1 & 1 & $\sqrt{2} / 2$ \\
$t_{i x}$ & 0 & 0 & 0 & 0 & 0 & 0 & 0 \\
$t_{i y}$ & 30.747 & -7.915 & $38.202+\delta_{y}$ & -7.915 & 30.747 & 0 & -12.7 \\
$t_{i z}$ & 0 & 0 & 0 & 0 & 0 & 0 & 0 \\
\hline $\mathbf{I}$ & $\mathbf{I}=\mathbf{8}$ & $\mathbf{I}=\mathbf{9}$ & $\mathbf{I}=\mathbf{1 0}$ & $\mathbf{I}=\mathbf{1 1}$ & $\mathbf{I}=\mathbf{1 2}$ & $\mathbf{I}=\mathbf{1 3}$ & $\mathbf{I}=\mathbf{1 4}$ \\
\hline \multirow{2}{*}{ Type } & Flat & Flat & Flat Mirror & Flat & Convex & Concave & CCD \\
& Refracting & Refracting & Refracting & Spherical & Spherical & \\
\hline $\mathrm{N}_{\mathrm{i}}$ & 1.515 & $1 / 1.515$ & Reflected & 1.515 & 1.515 & $1 / 1.515$ & \\
$\mathrm{R}_{\mathrm{i}}$ & 0 & 0 & 0 & 0 & 24.397 & 24.397 & 0 \\
$I_{i x}$ & -1 & -1 & -1 & -1 & -1 & -1 & -1 \\
$I_{i y}$ & 0 & 0 & 0 & 0 & 0 & 0 & 0 \\
$I_{i z}$ & 0 & 0 & 0 & 0 & 0 & 0 & 0 \\
$J_{i x}$ & 0 & 0 & 0 & 0 & 0 & 0 & 0 \\
$J_{i y}$ & 0 & 0 & $\sqrt{2} / 2$ & -1 & -1 & -1 & -1 \\
$I_{i z}$ & -1 & -1 & $-\sqrt{2} / 2$ & 0 & 0 & 0 & 0 \\
$K_{i x}$ & 0 & 0 & 0 & 0 & 0 & 0 & 0 \\
$K_{i y}$ & -1 & -1 & $-\sqrt{2} / 2$ & 0 & 0 & 0 & 0 \\
$K_{i z}$ & 0 & 0 & $-\sqrt{2} / 2$ & 1 & 1 & 1 & 1 \\
$t_{i x}$ & 0 & 0 & 0 & 0 & 0 & 0 & 0 \\
$t_{i y}$ & -12.7 & -12.7 & -12.7 & -25.4 & -56.147 & -17.485 & -63.602 \\
$t_{i z}$ & -12.7 & -31.9 & -38.1 & -44.6 & -44.6 & -44.6 & -44.6 \\
\hline & & & & & & & \\
\hline
\end{tabular}


Table 2. Parameters of the optical boundary coordinate transformation matrix for ray path II.

\begin{tabular}{ccccccccc}
\hline $\mathbf{I}$ & $\mathbf{I}=\mathbf{1}$ & $\mathbf{I}=\mathbf{2}$ & $\mathbf{I}=\mathbf{3}$ & $\mathbf{I}=\mathbf{4}$ & $\mathbf{I}=\mathbf{5}$ & $\mathbf{I}=\mathbf{6}$ & $\mathbf{I}=\mathbf{1 5}$ & $\mathbf{I}=\mathbf{1 6}$ \\
\hline Type & $\begin{array}{c}\text { Convex } \\
\text { Spherical }\end{array}$ & $\begin{array}{c}\text { Concave } \\
\text { Spherical }\end{array}$ & $\begin{array}{c}\text { Flat Mirror } \\
\text { (Sample) }\end{array}$ & $\begin{array}{c}\text { Convex } \\
\text { Spherical }\end{array}$ & $\begin{array}{c}\text { Concave } \\
\text { Spherical }\end{array}$ & $\begin{array}{c}\text { Flat } \\
\text { Refracting }\end{array}$ & $\begin{array}{c}\text { Flat } \\
\text { Refracting }\end{array}$ & CCD $_{\mathbf{2}}$ \\
\hline $\mathrm{N}_{\mathrm{i}}$ & $1 / 1.515$ & 1.515 & Reflected & $1 / 1.515$ & 1.515 & $1 / 1.515$ & 1.515 \\
$\mathrm{R}_{\mathrm{i}}$ & 24.397 & 24.397 & & 24.397 & 24.397 & & & \\
$I_{i x}$ & 1 & 1 & 1 & 1 & 1 & 1 & -1 & -1 \\
$I_{i y}$ & 0 & 0 & $\mathrm{~S} \omega_{z}$ & 0 & 0 & 0 & 0 & 0 \\
$I_{i z}$ & 0 & 0 & 0 & 0 & 0 & 0 & 0 & 0 \\
$J_{i x}$ & 0 & 0 & $-\mathrm{C} \omega_{x} \mathrm{~S} \omega_{z}$ & 0 & 0 & 0 & 0 & 0 \\
$J_{i y}$ & 1 & 1 & 1 & 1 & 1 & 1 & -1 & -1 \\
$J_{i z}$ & 0 & 0 & $\mathrm{C} \omega_{x} \mathrm{~S} \omega_{z}$ & 0 & 0 & 0 & 0 & 0 \\
$K_{i x}$ & 0 & 0 & $\mathrm{~S} \omega_{x} \mathrm{~S} \omega_{z}$ & 0 & 0 & 0 & 0 & 0 \\
$K_{i y}$ & 0 & 0 & $\mathrm{~S} \omega_{x} \mathrm{C} \omega_{z}$ & 0 & 0 & 0 & 0 & 0 \\
$K_{i z}$ & 1 & 1 & 1 & 1 & 1 & 1 & 1 & 1 \\
$t_{i x}$ & 0 & 0 & 0 & 0 & 0 & 0 & 0 & 0 \\
$t_{i y}$ & 30.747 & -7.915 & 38.202 & -7.915 & 30.747 & 0 & -25.4 & -49.426 \\
$t_{i z}$ & 0 & 0 & 0 & 0 & 0 & 0 & 0 & 0 \\
\hline
\end{tabular}

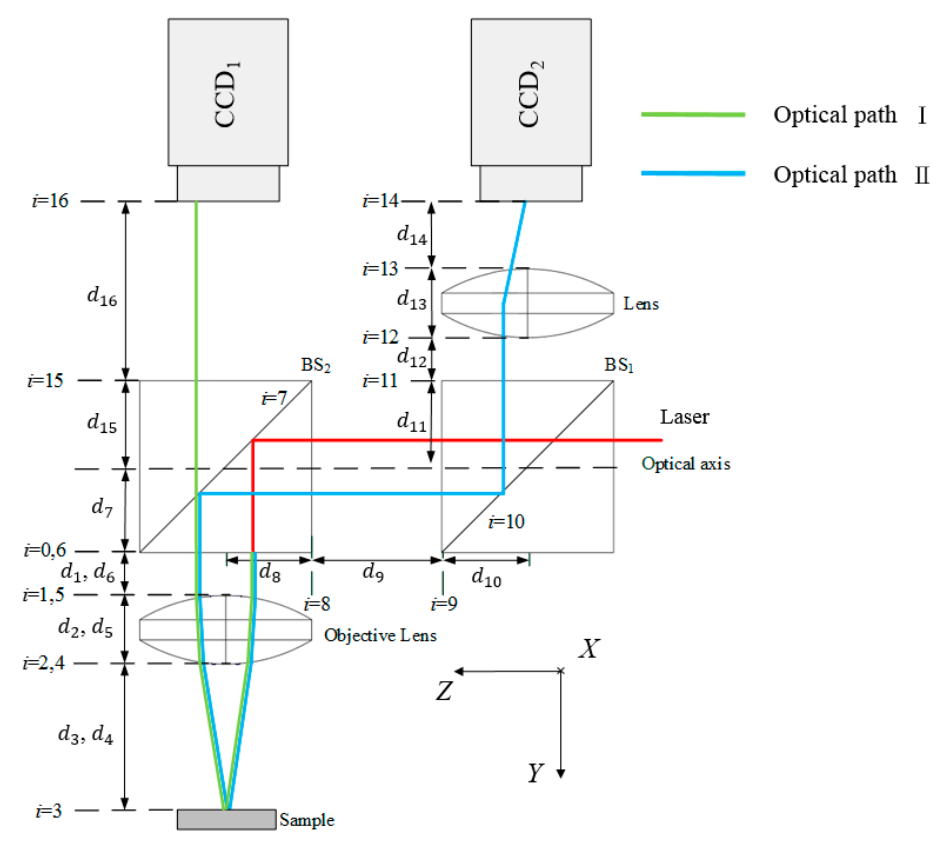

Figure 3. Definition of each optical boundary of the proposed measurement system.

\subsection{Incident Point and Unit Vector of Incident Laser Ray}

To trace the propagation of the laser ray in the proposed measurement system, the initial position

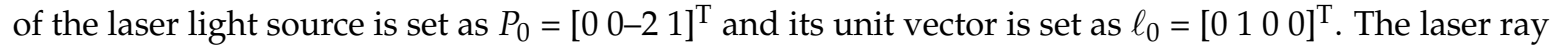
begins at an initial position that is along the unit vector but incident to the proposed measurement system. For the actual ray propagation in the proposed measurement system, the ray incident point on each optical boundary, the unit vectors of the reflected rays [14], and the unit vectors of the refracted rays are shown in Figure 4 and can be obtained from Equations (2) to (8). Equation (2) gives the expressions of the optical boundary ${ }^{i} \overline{r_{i}}$ and its unit normal vector ${ }^{i} \overline{n_{i}}$ with parameters $\alpha_{\mathrm{i}}$ and $\beta_{\mathrm{i}}$. Note that the non-negative parameter $\lambda_{i}$ represents the geometrical length from point $P_{i-1}$ to $P_{i}$. The \pm sign in Equation (3) indicates there may be two possible intersection points of this ray and a complete sphere. Clearly, only one of these points is useful, thus the appropriate sign must be chosen. The spherical coordinates, $\alpha_{\mathrm{i}}$ and $\beta_{\mathrm{i}}$, at the incidence point $P_{i}$ can be determined from Equation (6). 
Equations (7) and (8) define the $D_{i}$ and $E_{i}$ as the parameters in spherical and plane surface boundary situation, respectively.

The results of these calculations are described in Tables 3 and 4 . Tables 3 and 4 are the calculated parameters of the ray incident points and ray unit vectors of ray path I and II, respectively.

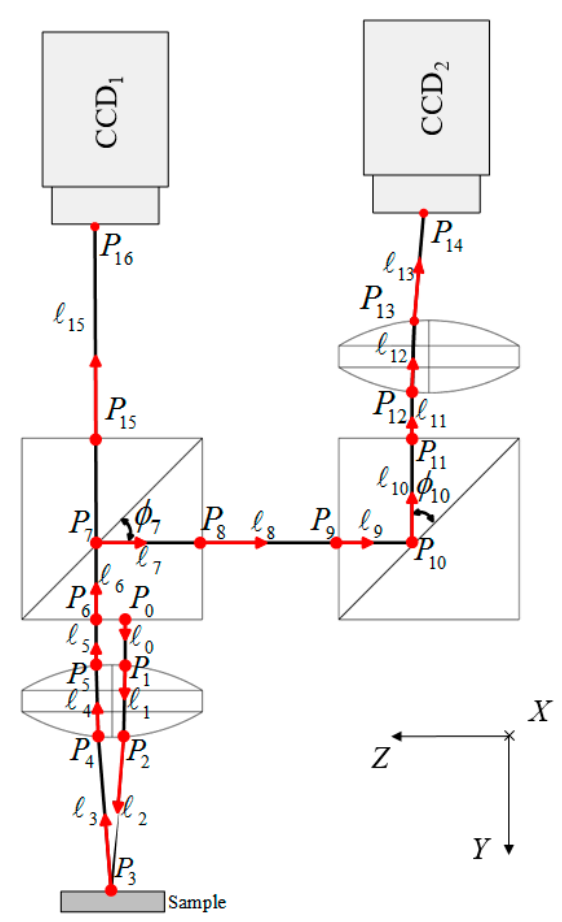

Figure 4. Definition of incident point and unit vectors of reflected and refracted rays.

Incident point

$$
\begin{gathered}
\bar{P}_{i}=\left[\begin{array}{c}
P_{i x} \\
P_{i y} \\
P_{i z} \\
1
\end{array}\right]=\left[\begin{array}{c}
P_{i-1 x}+\ell_{i-1 x} \lambda_{i} \\
P_{i-1 y}+\ell_{i-1 y} \lambda_{i} \\
P_{i-1 z}+\ell_{i-1 z} \lambda_{i} \\
1
\end{array}\right]=\left[\begin{array}{c}
\sigma_{i} \\
\rho_{i} \\
\tau_{i} \\
1
\end{array}\right]=\left[\begin{array}{c}
\left|R_{i}\right| \mathrm{C} \beta_{i} \mathrm{C} \alpha_{i} \\
\left|R_{i}\right| \mathrm{C} \beta_{i} \mathrm{~S} \alpha_{i} \\
\left|R_{i}\right| \mathrm{S} \beta_{i} \\
1
\end{array}\right] \\
\lambda_{i}=-D_{i} \pm \sqrt{D_{i}^{2}-E_{i}}
\end{gathered}
$$

Boundary equations

Spherical surface boundary:

$$
\begin{aligned}
& D_{i}=\rho_{i}\left(-\ell_{i-1 y}\right)+\tau_{i} \ell_{i-1 z}+P_{i-1 x} \ell_{i-1 x}+P_{i-1 y} \ell_{i-1 y}+P_{i-1 z} \ell_{i-1 z} \\
& E_{i}=P_{i-1 x}^{2}+P_{i-1 y}^{2}+P_{i-1 z}^{2}+\rho_{i}^{2}+\tau_{i}^{2}-R_{i}^{2}-2\left(\rho_{i} P_{i-1 y}+\tau_{i} P_{i-1 z}\right)
\end{aligned}
$$

Plane surface boundary:

$$
\begin{gathered}
D_{i}=n_{i x} P_{i-1 x}+n_{i y} P_{i-1 y}+n_{i z} P_{i-1 z}+e_{i} \\
E_{i}=n_{i x} \ell_{i-1 x}+n_{i y} \ell_{i-1 y}+n_{i z} \ell_{i-1 z}
\end{gathered}
$$

For the purpose of tracing the reflected or refracted ray incidents at the boundary surface, one needs the incidence angle $\theta_{i}\left(0^{\circ} \leqslant \theta_{i} \leqslant 90^{\circ}\right)$, which is determined by the dot product of $\bar{\ell}_{i-1}$ and the active unit normal vector $\bar{n}_{\mathrm{i}}$. Then the ray unit directional vector $\bar{\ell}_{i}$ can be obtained by the refraction and reflection law of optics, and Equations (7) and (8) show the calculated and simplified 
result of them. Note that $N_{i}$ is the index of refraction or reflection defined by the Snell's law. More details about the skew-ray tracing method derivation process are given in publications by Lin $[14,15]$.

$$
\begin{aligned}
& C \theta_{i}=\left|\bar{\ell}_{i-1} \bullet \bar{n}_{i}\right|=-\bar{\ell}_{i-1} \bullet \bar{n}_{i} \\
& \alpha_{i}=\operatorname{atan} 2\left(P_{i-1 y}+\ell_{i-1 y} \lambda_{i}+\rho_{i}, P_{i-1 x}+\ell_{i-1 x} \lambda_{i}\right) \\
& \beta_{i}=\operatorname{atan} 2\left(P_{i-1 z}+\ell_{i-1 z} \lambda_{i}+\tau_{i}, \sqrt{\left(P_{i-1 x}+\ell_{i-1 x} \lambda_{i}\right)^{2}+\left(P_{i-1 y}+\ell_{i-1 y} \lambda_{i}+\rho_{i}\right)^{2}}\right)
\end{aligned}
$$

\begin{tabular}{|c|c|c|c|c|c|c|c|}
\hline I & $\mathrm{I}=1$ & $\mathrm{I}=2$ & $I=3$ & $\mathrm{I}=4$ & $I=5$ & $I=6$ & $\mathrm{I}=7$ \\
\hline Type & $\begin{array}{l}\text { Convex } \\
\text { Spherical }\end{array}$ & $\begin{array}{l}\text { Concave } \\
\text { Spherical }\end{array}$ & $\begin{array}{l}\text { Flat Mirror } \\
\text { (Sample) }\end{array}$ & $\begin{array}{l}\text { Convex } \\
\text { Spherical }\end{array}$ & Concave Spherical & $\begin{array}{c}\text { Flat } \\
\text { Refracting }\end{array}$ & $\begin{array}{c}\text { Flat } \\
\text { Refracting }\end{array}$ \\
\hline $\mathrm{N}_{\mathrm{i}}$ & $1 / 1.515$ & 1.515 & Reflected & $1 / 1.515$ & 1.515 & $1 / 1.515$ & Reflected \\
\hline$\phi_{i}$ & 0 & 0 & 0 & 0 & 0 & 0 & $\pi / 4$ \\
\hline$n_{i x}$ & $\mathrm{C} \beta_{1} \mathrm{C} \alpha_{1}$ & $\mathrm{C} \beta_{1} \mathrm{C} \alpha_{1}$ & $-\mathrm{C} \omega_{x} \mathrm{~S} \omega_{z}$ & $\mathrm{C} \beta_{4} \mathrm{C} \alpha_{4}$ & $\mathrm{C} \beta_{5} \mathrm{C} \alpha_{5}$ & 0 & 0 \\
\hline$n_{i y}$ & $C \beta_{1} C \alpha_{1}$ & $\mathrm{C} \beta_{1} \mathrm{C} \alpha_{1}$ & $\mathrm{C} \omega_{x} \mathrm{C} \omega_{z}$ & $\mathrm{C} \beta_{4} \mathrm{C} \alpha_{4}$ & $\mathrm{C} \beta_{5} \mathrm{C} \alpha_{5}$ & 1 & $\mathrm{~S} \phi_{7}$ \\
\hline$n_{i z}$ & $\mathrm{~S} \beta_{1}$ & $\mathrm{~S} \beta_{1}$ & $\mathrm{~S} \omega_{x}$ & $\mathrm{~S} \beta_{4}$ & $\mathrm{~S} \beta_{5}$ & 0 & $-\mathrm{C} \phi_{7}$ \\
\hline$\rho_{i}$ & $-\left(\mathrm{d}_{1}+\mathrm{R}\right)$ & $\mathrm{R}-\left(\mathrm{d}_{1}+\mathrm{d}_{2}\right)$ & 0 & $\mathrm{R}-\left(\mathrm{d}_{1}+\mathrm{d}_{2}\right)$ & $-\left(\mathrm{d}_{1}+\mathrm{R}\right)$ & 0 & 0 \\
\hline$\tau_{i}$ & 0 & 0 & 0 & 0 & 0 & 0 & 0 \\
\hline$e_{i}$ & Sphere & Sphere & $-\left(\mathrm{d}_{1}+\mathrm{d}_{2}+\mathrm{d}_{3}\right)$ & Sphere & Sphere & 0 & $-\mathrm{d}_{7} \mathrm{~S} \phi_{7}$ \\
\hline I & $\mathrm{I}=8$ & $I=9$ & $\mathrm{I}=10$ & $\mathrm{I}=\mathbf{1 1}$ & $\mathrm{I}=12$ & $I=13$ & $\mathrm{I}=14$ \\
\hline Type & $\begin{array}{c}\text { Flat } \\
\text { Refracting }\end{array}$ & $\begin{array}{c}\text { Flat } \\
\text { Refracting }\end{array}$ & Flat Mirror & $\begin{array}{l}\text { Flat Mirror } \\
\text { (Sample) }\end{array}$ & Convex Spherical & $\begin{array}{l}\text { Concave } \\
\text { Spherical }\end{array}$ & $\mathrm{CCD}_{1}$ \\
\hline $\mathrm{N}_{\mathrm{i}}$ & 1.515 & 1.515 & Reflected & 1.515 & $1 / 1.515$ & 1.515 & $1 / 1.515$ \\
\hline$\phi_{i}$ & 0 & 0 & $3 \pi / 4$ & 0 & 0 & 0 & \\
\hline$n_{i x}$ & 0 & 0 & 0 & 0 & $\mathrm{C} \beta_{5} \mathrm{C} \alpha_{5}$ & 0 & 1 \\
\hline$n_{i y}$ & 0 & 0 & $\mathrm{C} \phi_{10}$ & 1 & $C \beta_{5} C \alpha_{5}$ & 1 & 0 \\
\hline$n_{i z}$ & 1 & 1 & $\mathrm{~S} \phi_{10}$ & 0 & $\mathrm{~S} \beta_{5}$ & 0 & 0 \\
\hline$\rho_{i}$ & 0 & 0 & 0 & 0 & $\begin{array}{c}\mathrm{d}_{7} \mathrm{~S} 2 \phi_{10}-\mathrm{d}_{8} \mathrm{C} 2 \phi_{10} \\
\quad+\mathrm{d}_{11}+\mathrm{d}_{12}+\mathrm{R}\end{array}$ & $\begin{array}{l}\mathrm{d}_{7}+\mathrm{d}_{11}+\mathrm{d}_{12} \\
\quad+\mathrm{d}_{13}-\mathrm{R}\end{array}$ & 0 \\
\hline$\tau_{i}$ & 0 & 1 & 0 & 0 & $\begin{array}{c}\mathrm{d}_{7} \mathrm{C} 2 \phi_{10}+\mathrm{d}_{8} \mathrm{~S} 2 \phi_{10} \\
+\mathrm{d}_{10}+\mathrm{d}_{9}\end{array}$ & $\mathrm{~d}_{8}+\mathrm{d}_{9}+\mathrm{d}_{10}$ & 1 \\
\hline$e_{i}$ & $-\mathrm{d}_{8}$ & $-\left(d_{8}+d_{9}\right)$ & $\begin{array}{c}\left(\mathrm{d}_{8}+\mathrm{d}_{9}+\mathrm{d}_{10}\right) \mathrm{S} \phi_{10} \\
-\mathrm{d}_{7} C \phi_{10}\end{array}$ & $\begin{array}{c}\mathrm{d}_{8} \mathrm{C} 2 \phi_{10}- \\
\mathrm{d}_{11}-\mathrm{d}_{7} \mathrm{~S} 2 \phi_{10}\end{array}$ & Sphere & Sphere & $\begin{array}{c}-\mathrm{d}_{7}-\mathrm{d}_{11} \\
-\mathrm{d}_{12}- \\
\mathrm{d}_{13}-\mathrm{d}_{14}\end{array}$ \\
\hline
\end{tabular}

Table 3. Ray incident points and ray unit vector parameters of ray path I.

The unit vector of the reflected ray:

$$
\bar{\ell}_{i}=\left[\begin{array}{c}
\ell_{i x} \\
\ell_{i y} \\
\ell_{i z} \\
0
\end{array}\right]=\left[\begin{array}{c}
\ell_{i-1 x}+2 C \theta_{i} n_{i x} \\
\ell_{i-1 y}+2 C \theta_{i} n_{i y} \\
\ell_{i-1 z}+2 C \theta_{i} n_{i z} \\
0
\end{array}\right]=\bar{\ell}_{i-1}+2 C \theta_{i} n_{i}
$$

The unit vector of the refracted ray:

$$
\bar{\ell}_{i}=\left[\begin{array}{c}
\ell_{i x} \\
\ell_{i y} \\
\ell_{i z} \\
0
\end{array}\right]=\left[\begin{array}{c}
-n_{i x} \sqrt{1-N_{i}^{2}+\left(N_{i} C \theta_{i}\right)^{2}}+N_{i}\left(\ell_{i-1 x}+n_{i x} C \theta_{i}\right) \\
-n_{i y} \sqrt{1-N_{i}^{2}+\left(N_{i} C \theta_{i}\right)^{2}}+N_{i}\left(\ell_{i-1 y}+n_{i y} C \theta_{i}\right) \\
-n_{i z} \sqrt{1-N_{i}^{2}+\left(N_{i} C \theta_{i}\right)^{2}}+N_{i}\left(\ell_{i-1 z}+n_{i z} C \theta_{i}\right) \\
0
\end{array}\right]=\left(N_{i} C \theta_{i}-\sqrt{1-N_{i}^{2}+\left(N_{i} C \theta_{i}\right)^{2}}\right) \overline{n_{i}}+N_{i} \bar{\ell}_{i-1}
$$

Sequentially tracing the ray incident points on each optical boundary gives the coordinates of the spots on the two CCDs. Because $\omega_{x}, \omega_{z}$ and $\delta_{y}$ are generally very small, a first-order Taylor series expanded about $\omega_{x}=\omega_{z}=\delta_{y}=0$ can be used to obtain a first-order equation of the proposed system measurement. Then, substituting into the equation the parameters for the actual sensor position and measurement system, such as the system internal unit distance $d_{i}$ and the lens surface radius $R_{i}$, the system measurement Equation (9) of the spot coordinates on the two CCDs can be obtained: 


$$
\left\{\begin{array}{l}
P_{14 x}=215.147\left(3.44801-0.155909 \delta_{y}+0.780777 \omega_{\mathrm{x}}\right) \\
P_{14 z}=214.876\left(2.69362+0.0402449 \omega_{\mathrm{z}}\right) \\
P_{16 x}=215.147\left(3.47192+0.464506 \delta_{y}+48.5221 \omega_{\mathrm{x}}\right) \\
P_{16 z}=214.876\left(2.43596-50.5223 \omega_{\mathrm{z}}\right)
\end{array}\right.
$$

Equation (10) is the result of a reverse derivation of solving Equation (9). Equation (10) gives a set of linear equations that gives accurate sample slanting angles $\left(\omega_{x}, \omega_{z}\right)$ and distance $\left(\delta_{y}\right)$ from the spot center locations on the CCDs:

$$
\begin{aligned}
& \omega_{x}=-0.27030+0.00027 P_{14 x}+0.00009 P_{16 x} \\
& \omega_{z}=0.04822-0.00009 P_{16 z} \\
& \delta_{y}=20.76186-0.02845 P_{14 x}+0.00046 P_{16 x}
\end{aligned}
$$

\begin{tabular}{|c|c|c|c|c|c|c|c|c|}
\hline $\mathbf{I}$ & $\mathrm{I}=\mathbf{1}$ & $I=2$ & $\mathbf{I}=3$ & $I=4$ & $I=5$ & $I=6$ & $I=15$ & $I=16$ \\
\hline Type & $\begin{array}{l}\text { Convex } \\
\text { Spherical }\end{array}$ & $\begin{array}{l}\text { Concave } \\
\text { Spherical }\end{array}$ & $\begin{array}{c}\text { Flat Mirror } \\
\text { (Sample) }\end{array}$ & $\begin{array}{l}\text { Convex } \\
\text { Spherical }\end{array}$ & $\begin{array}{l}\text { Concave } \\
\text { Spherical }\end{array}$ & $\begin{array}{c}\text { Flat } \\
\text { Refracting }\end{array}$ & $\begin{array}{c}\text { Flat } \\
\text { Refracting }\end{array}$ & $\mathrm{CCD}_{2}$ \\
\hline $\mathrm{N}_{\mathrm{i}}$ & $1 / 1.515$ & 1.515 & Reflected & $1 / 1.515$ & 1.515 & $1 / 1.515$ & 1.515 & 1.515 \\
\hline$\phi_{i}$ & 0 & 0 & 0 & 0 & 0 & 0 & 0 & 0 \\
\hline$n_{i x}$ & $\mathrm{C} \beta_{1} \mathrm{C} \alpha_{1}$ & $\mathrm{C} \beta_{2} \mathrm{C} \alpha_{2}$ & $-\mathrm{C} \omega_{x} \mathrm{~S} \omega_{z}$ & $\mathrm{C} \beta_{4} \mathrm{C} \alpha_{4}$ & $C \beta_{5} C \alpha_{5}$ & 0 & 0 & 0 \\
\hline$n_{i y}$ & $\mathrm{C} \beta_{1} \mathrm{C} \alpha_{1}$ & $\mathrm{C} \beta_{2} \mathrm{C} \alpha_{2}$ & $\mathrm{C} \omega_{x} \mathrm{C} \omega_{z}$ & $\mathrm{C} \beta_{4} \mathrm{C} \alpha_{4}$ & $\mathrm{C} \beta_{5} \mathrm{C} \alpha_{5}$ & 1 & 1 & 1 \\
\hline$n_{i z}$ & $\mathrm{~S} \beta_{1}$ & $\mathrm{~S} \beta_{2}$ & $\mathrm{~S} \omega_{x}$ & $\mathrm{~S} \beta_{4}$ & $\mathrm{~S} \beta_{5}$ & 0 & 0 & 0 \\
\hline$\rho_{i}$ & $-\left(\mathrm{d}_{1}+\mathrm{R}\right)$ & $\mathrm{R}-\left(\mathrm{d}_{1}+\mathrm{d}_{2}\right)$ & 0 & $\mathrm{R}-\left(\mathrm{d}_{1}+\mathrm{d}_{2}\right)$ & $-\left(\mathrm{d}_{1}+\mathrm{R}\right)$ & 0 & 0 & 0 \\
\hline$\tau_{i}$ & 0 & 0 & 0 & 0 & 0 & 0 & 0 & 0 \\
\hline$e_{i}$ & Sphere & Sphere & $-\left(\mathrm{d}_{1}+\mathrm{d}_{2}+\mathrm{d}_{3}\right)$ & Sphere & Sphere & 0 & $-\mathrm{d}_{15}$ & $-\mathrm{d}_{15}-\mathrm{d}_{16}$ \\
\hline
\end{tabular}

Table 4. Ray incident points and ray unit vector parameters of ray path II.

\section{Experimental Results and Discussion}

Figure 5 shows the actual design of the proposed measurement system on an optical bench.

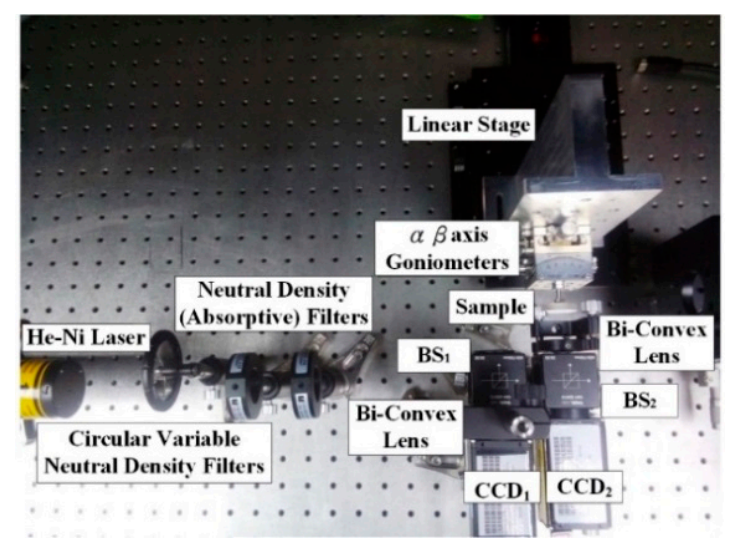

Figure 5. Photograph of the laboratory-built prototype.

The experimental set-up includes placing the sample on a dual $(\alpha-\beta)$ axis goniometer that rotates about the $\alpha$ and $\beta$ axes with a resolution of $0.1^{\circ}$. LabVIEW (National Instruments Co., Mopac Expwy Austin, TX, USA ) is used to control the linear stage (one step of $1 \mathrm{~lm}$, HF-KP053-B series, Chiuan Yan Tech, Changhua, Taiwan) to induce mono-dimensional movements of the sample (i.e., distance $\left.\left(\delta_{y}\right)\right)$, and a He-Ne Laser is used as the system light source $(\lambda=632 \mathrm{~nm}, 2 \mathrm{~mW})$.

\subsection{Part 1}

The measurement results of the sample distance $\left(\delta_{y}\right)$ and slanting angles $\left(\omega_{x}, \omega_{z}\right)$ are verified individually. When verifying the measurement results of the distance $\left(\delta_{y}\right)$, the sample is driven by 
a linear stage along the $y$-direction with both slanting angles $\left(\omega_{x}, \omega_{z}\right)$ set to $0^{\circ}$. The measurement range of the distance is $-1 \sim+1 \mathrm{~mm}$. When verifying the measurement results of the flat sample slanting angle $\omega_{x}$ using a dual axis goniometer, the sample distance $\left(\delta_{y}\right)$ is fixed to $0 \mathrm{~mm}$, and the slanting angle $\omega_{z}$ is set to $0^{\circ}$. The measurement range of the slanting angle is $-1^{\circ} \sim+1^{\circ}$. The verification method of the measurement results of the flat sample slanting angle $\omega_{z}$ is similar to that of $\omega_{x}$. The verification results are shown in Figure 6. All measurement points in Figure 6 originate from simultaneous images captured by two CCDs. The sampling frequency is one image per second, and 20 images are captured per measured point. Then, the average values of the sample distance $\left(\delta_{y}\right)$ and slanting angles $\left(\omega_{x}, \omega_{z}\right)$ are calculated using the results of the mathematical derivations in the previous section. It is observed that the measured errors of the slanting angles $\left(\omega_{x}, \omega_{z}\right)$ and the distance $\left(\delta_{y}\right)$ are $0.009^{\circ}$ and $6 \mu \mathrm{m}$, respectively. In other words, the measured linearity of the slanting angles $\left(\omega_{x}, \omega_{z}\right)$ and the distance $\left(\delta_{y}\right)$ is $0.45 \%$ and $0.3 \%$, respectively.

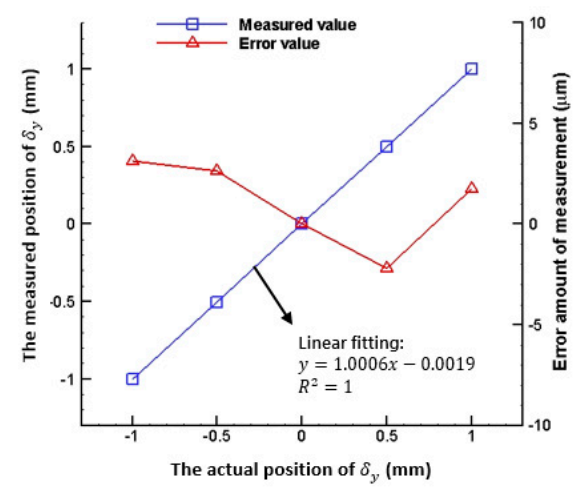

(a)

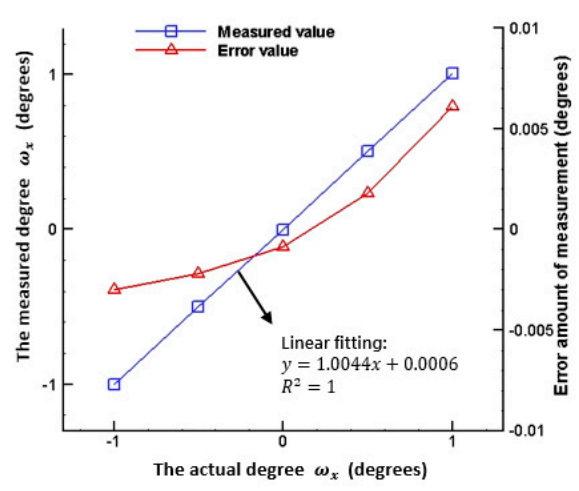

(b)

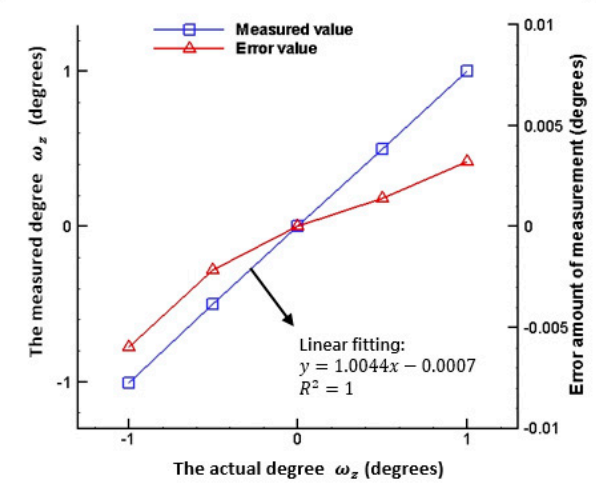

(c)

Figure 6. Verification of experimental results: (a) distance $\left(\delta_{y}\right)$; (b) slanting angle $\left(\omega_{x}\right)$; and (c) slanting angle $\left(\omega_{z}\right)$.

\subsection{Part 2}

This part would show the complex measurement results of the sample distance $\left(\delta_{y}\right)$ and slanting angles $\left(\omega_{x}, \omega_{z}\right)$. However the possible permutations of the sample distance $\left(\delta_{y}\right)$ and slanting angles $\left(\omega_{x}, \omega_{z}\right)$ multiply towards infinity. This part would verify the measurement results of the distance $\left(\delta_{y}\right)$ under the setting of slanting angles $\left(\omega_{x}, \omega_{z}\right)$ that are equal to $\left(2^{\circ}, 2^{\circ}\right),\left(-2^{\circ},-2^{\circ}\right),\left(-2^{\circ}, 2^{\circ}\right),\left(2^{\circ},-2^{\circ}\right)$. The purpose of this experiment is to find the limit of the laboratory-built prototype.

The maximum measurement range of the distance $\left(\delta_{y}\right)$ is $-4 \sim+4 \mathrm{~mm}$ and each measured point is $1 \mathrm{~mm}$ apart. The sampling frequency is one image per second, and 20 images are captured per measured point. All measurement points in Figure 7 originate from simultaneous images captured by two CCDs. Figure 7 shows the experimental results are very similar to the simulation results on two CCDs. 

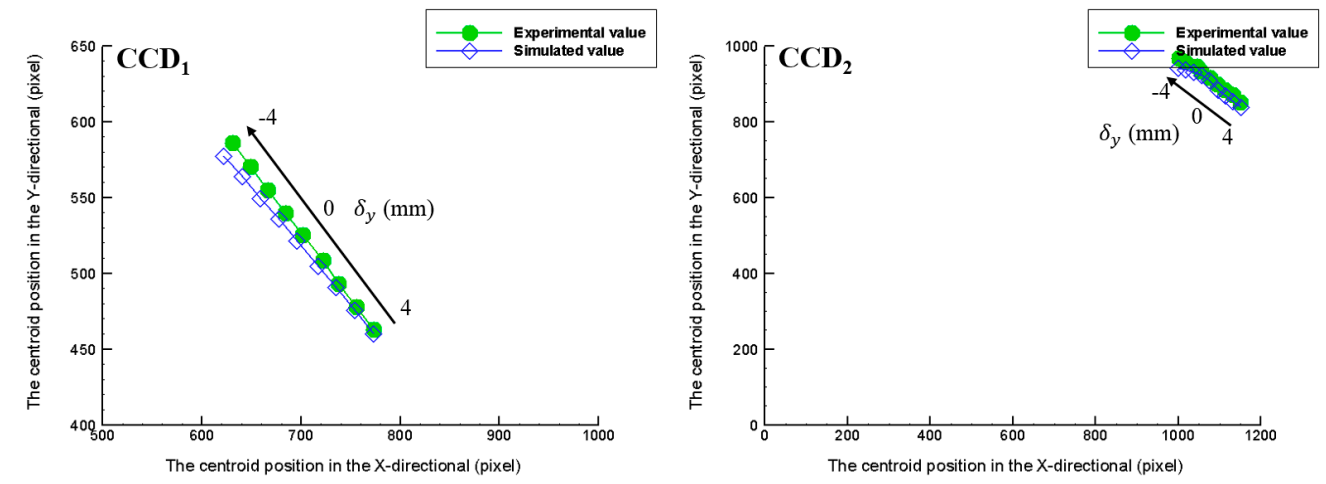

(a)
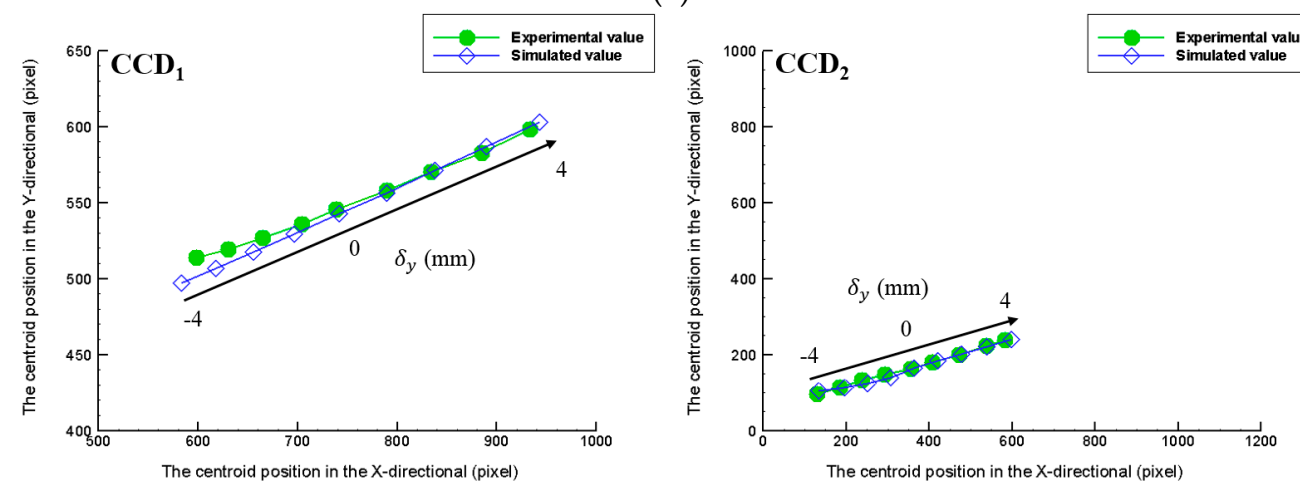

(b)
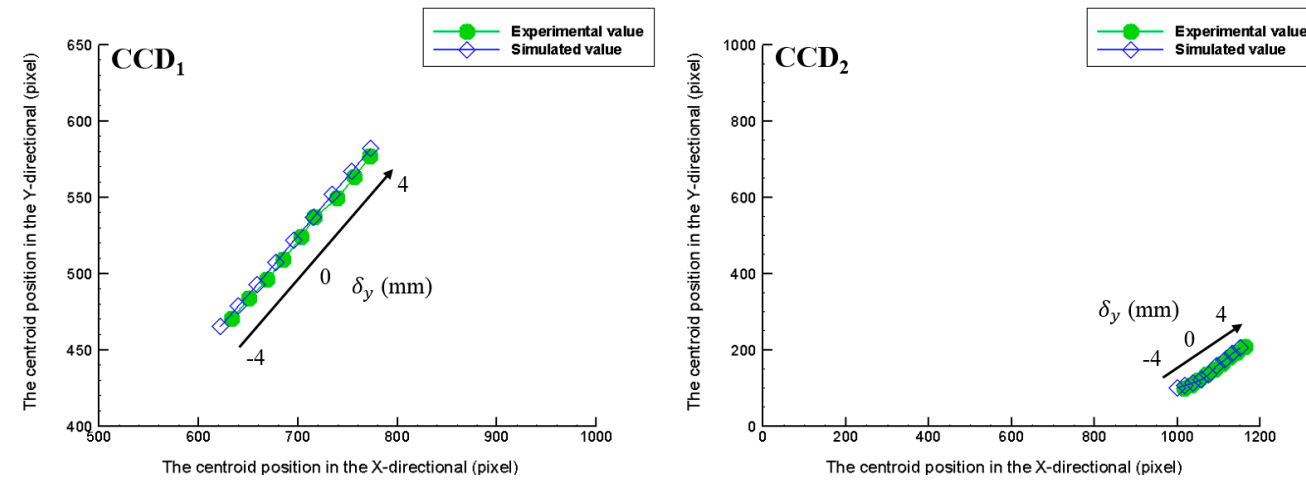

(c)
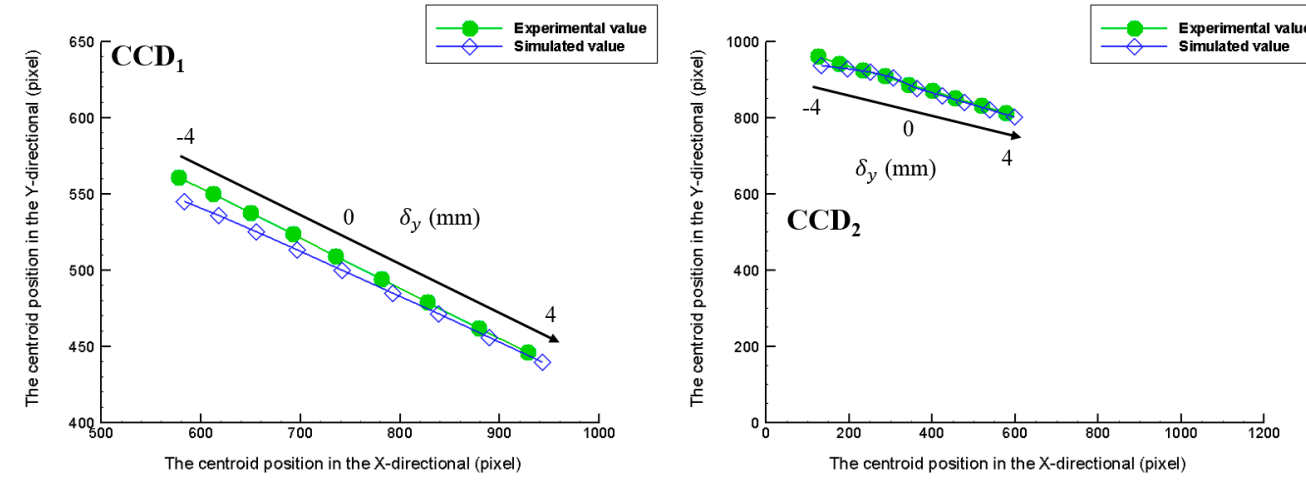

(d)

Figure 7. Verification of the distance $\left(\delta_{y}\right)$ results with the slanting angles $\left(\omega_{x}, \omega_{z}\right):(\mathbf{a})\left(2^{\circ}, 2^{\circ}\right)$, (b) $\left(-2^{\circ},-2^{\circ}\right),\left(\right.$ c) $\left(2^{\circ},-2^{\circ}\right)$, and $($ d $)\left(-2^{\circ}, 2^{\circ}\right)$. 


\section{System Stability Test}

The stability test involves first placing the proposed measurement system in a stable environment for at least $15 \mathrm{~min}$ to regulate the temperature of the system. Then, the sample is adjusted to a fixed orientation and distance (e.g., distance $\delta_{y}=0 \mathrm{~mm}$; slanting angles $\omega_{x}=\omega_{z}=0^{\circ}$ ), and the system is sampled continuously for $6 \mathrm{~min}$. The results of the system stability test are shown in Figure 8. As seen, system stability is achieved within $6 \mathrm{~min}$ at a distance and inline angle of $3 \mu \mathrm{m}$ and $0.01^{\circ}$, respectively. However the measured accuracy of the proposed measurement system is not only influenced by the laser beam instability but also by misalignments, aberrations, CCD sensitivity, etc. and can be enhanced by using high quality laser and close-loop with a feedback signal methods [16,17]. As a result, in the future, these factors will need to be considered and optimized to reduce system errors and improve measured accuracy. Also we will produce modified physics model for real applications in industry and we must overcome some additional challenges. In our plan, we will combine our previous study to improve robustness toward geometrical fluctuations of the laser beam of the proposed measurement system and analyze accuracy influences by the components position error, misalignments, aberrations, quantization errors, vibrations, and laser non-stability effect, etc.

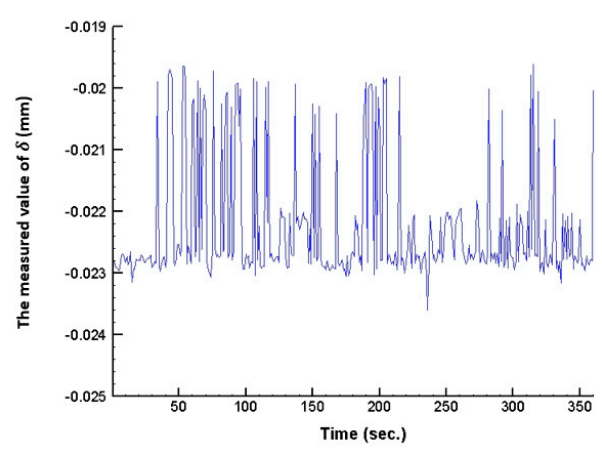

(a)

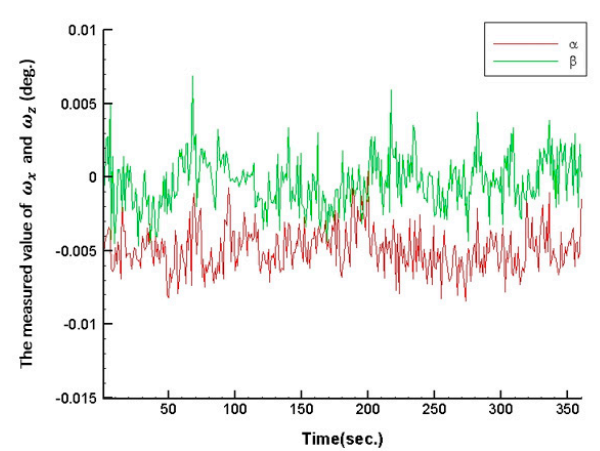

(b)

Figure 8. Results of proposed measurement system stability test: (a) distance $\left(\delta_{y}\right)$ and (b) slanting angles $\left(\omega_{x}, \omega_{z}\right)$.

\section{Comparison of Proposed Measurement System and Automatic Collimator}

We performed a comparison test using a commercially available automatic collimator (H400-C050, measurement range: $\pm 0.5^{\circ}$, Suruga Seiki Co., Shizuoka, Japan) and the proposed measurement system to measure the slanting angles of the sample $\left(\omega_{x}, \omega_{z}\right)$. As shown in Figure 9, the sample is placed on a dual axis goniometer that is installed on a linear stage. The proposed measurement system and the commercially available automatic collimator are configured alongside each other to monitor the slanting angles $\left(\omega_{x}, \omega_{z}\right)$ of the sample on the linear stage simultaneously. The linear stage is driven so that the automatic collimator and the proposed measurement system can simultaneously measure the sample slanting angle information in scanning mode. Figure 10 shows the results of comparing the proposed measurement system and the conventional automatic collimator. Using the dual axis goniometer to set the sample slanting angles as $\omega_{x}=0.5^{\circ}$ and $\omega_{z}=0^{\circ}$, one measurement is recorded for every $1 \mathrm{~mm}$ movement of the linear stage. Each recorded point is the averaged result of 10 separate measurements. The experimental results have shown that the proposed measurement system has excellent performance and its practicability, compared to the conventional automatic collimator. 


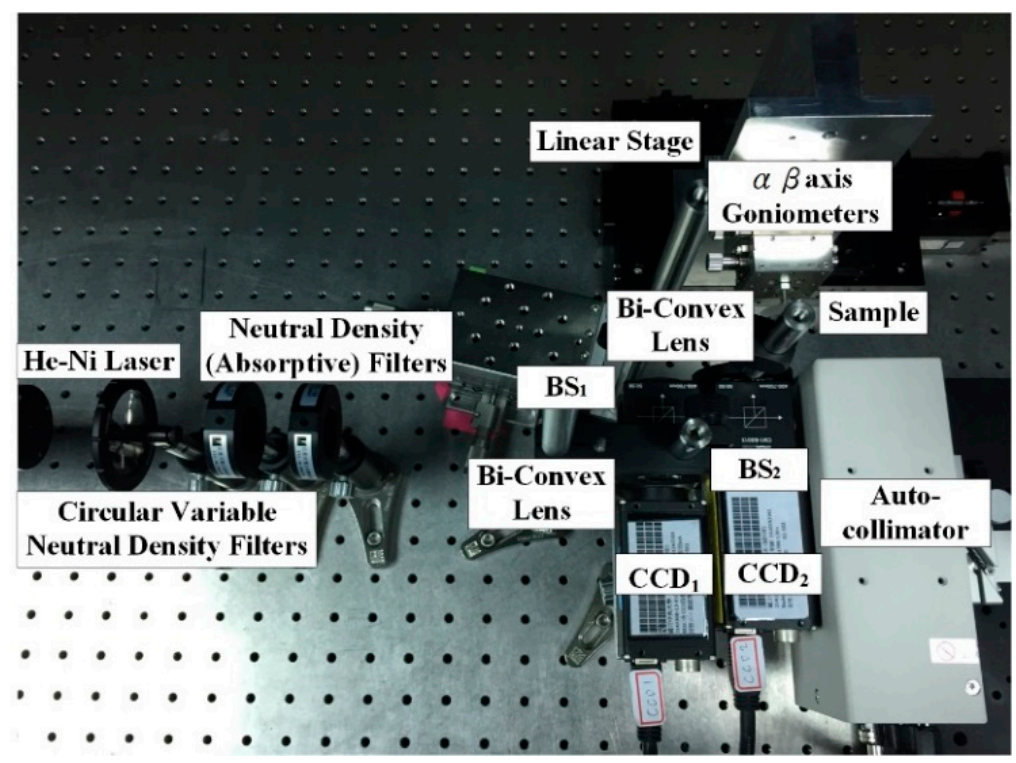

Figure 9. Set-up of the proposed measurement system and automatic collimator comparison test.

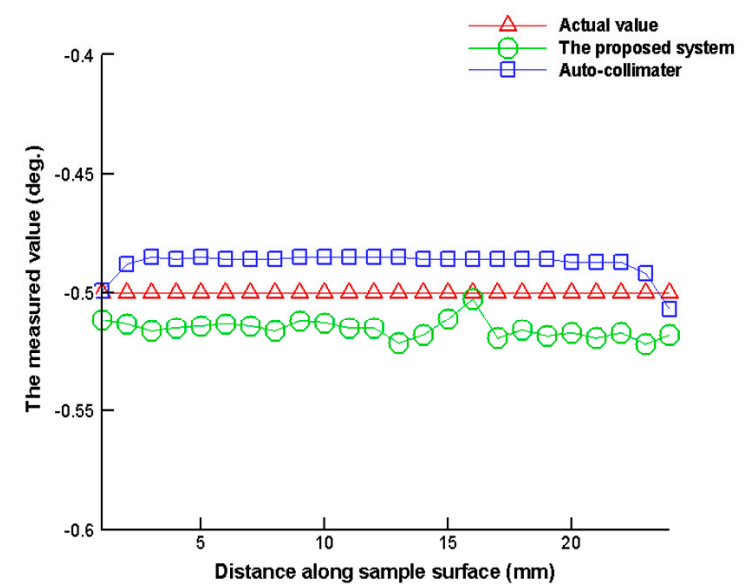

(a)

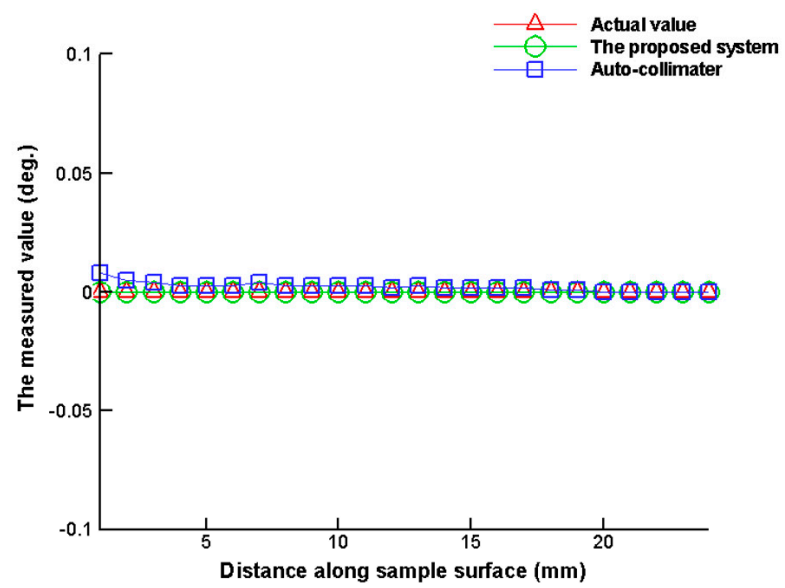

(b)

Figure 10. Comparative test results of proposed measurement system and automatic collimator: (a) $\omega_{x}$ experimental results and (b) $\omega_{z}$ experimental results.

\section{Conclusions}

An optical non-contacting measurement system has been successfully developed in this study. This system can measure workpiece samples with a slanting angle and simultaneously measure the sample distance and its orientation. The optical simulation software Zemax has been used to perform a feasibility analysis of this system. Additionally, a model of the proposed measurement system has been established using a HTM. The skew-ray tracing method is employed to trace the actual ray propagation path to obtain the system measurement equations. Finally, a laboratory-built prototype is constructed on an optical bench to verify the performance of the proposed measurement system. The orientation and distance measurement experiments show that the measuring ranges for the angles and distance are $\pm 1^{\circ}$ and $\pm 1 \mathrm{~mm}$, respectively, and the measured errors of the angles and the distance are $0.009^{\circ}$ and $6 \mu \mathrm{m}$, respectively. In other words, the measured linearity of the slanting angles $\left(\omega_{x}, \omega_{z}\right)$ and the distance $\left(\delta_{y}\right)$ is $0.45 \%$ and $0.3 \%$, respectively. 
Acknowledgments: The authors gratefully acknowledge the financial support provided to this study by the Ministry of Science and Technology of Taiwan under Grant Nos. MOST 103-2221-E-194-006-MY3 and 105-2218-E-194-004.

Author Contributions: Yen-Sheng Huang proposed the original idea, carried out the simulation and performed acquisition of the experimental data. Yu-Ta Chen implemented the mathematical model and analyzed the experiment results. Chien-Sheng Liu proposed the research direction and gave the technical support and conceptual advice. Yu-Ta Chen and Chien-Sheng Liu drafted, organized, revised, and proofed the paper.

Conflicts of Interest: The authors declare no conflict of interest.

\section{References}

1. Du, Z.; Wu, Z.; Yang, J. Error ellipsoid analysis for the diameter measurement of cylindroid components using a laser radar measurement system. Sensors 2016, 16, 714. [CrossRef] [PubMed]

2. Kaloshin, G.; Lukin, I. Interferometric laser scanner for direction determination. Sensors 2016, 16, 130. [CrossRef] [PubMed]

3. Liu, C.S.; Jiang, S.H. A novel laser displacement sensor with improved robustness toward geometrical fluctuations of the laser beam. Meas. Sci. Technol. 2013, 24, 105101. [CrossRef]

4. Lu, S.H.; Hua, H. Imaging properties of extended depth of field microscopy through single-shot focus scanning. Opt. Express 2015, 23, 10714-10731. [CrossRef] [PubMed]

5. Liu, C.S.; Jiang, S.H. Precise autofocusing microscope with rapid response. Opt. Lasers Eng. 2015, 66, $294-300$. [CrossRef]

6. Liu, C.S.; Jiang, S.H. Design and experimental validation of novel enhanced-performance autofocusing microscope. Appl. Phys. B 2014, 117, 1161-1171. [CrossRef]

7. Liu, C.S.; Wang, Z.Y.; Chang, Y.C. Design and characterization of high-performance autofocusing microscope with zoom in/out functions. Appl. Phys. B 2015, 121, 69-80. [CrossRef]

8. Makai, J.P. Simultaneous spatial and angular positioning of plane specular samples by a novel double beam triangulation probe with full auto-compensation. Opt. Lasers Eng. 2016, 77, 137-142. [CrossRef]

9. Lee, S.J.; Chang, D.Y. A laser sensor with multiple detectors for freeform surface digitization. Int. J. Adv. Manuf. Technol. 2007, 31, 1181-1190. [CrossRef]

10. Shiou, F.J.; Lee, R.T. Opto-Electronic Detector for Distance and Slanting Direction Measurement of a Surface. TW Patent I246585, 1 January 2006.

11. Gao, W.; Huang, P.S.; Yamada, T.; Kiyono, S. A compact and sensitive two-dimensional angle probe for flatness measurement of large silicon wafers. Precis. Eng. 2002, 26, 396-404. [CrossRef]

12. Lin, P.D.; Sung, C.K. Matrix-based paraxial skew ray-tracing in 3D systems with non-coplanar optical axis. Opt. Int. J. Light Electron Opt. 2006, 117, 329-340. [CrossRef]

13. Chen, C.J.; Lin, P.D.; Jywe, W.Y. An optoelectronic measurement system for measuring 6-degree-of-freedom motion error of rotary parts. Opt. Express 2007, 15, 14601-14617. [CrossRef] [PubMed]

14. Lin, P.D. Derivative matrices of a skew ray for spherical boundary surfaces and their applications in system analysis and design. Appl. Opt. 2014, 53, 3085-3100. [CrossRef] [PubMed]

15. Lin, P.D. New Computation Methods for Geometrical Optics; Springer: Singapore, 2013.

16. Grover, G.; Mohrman, W.; Piestun, R. Real-time adaptive drift correction for super-resolution localization microscopy. Opt. Express 2015, 23, 23887-23898. [CrossRef] [PubMed]

17. Rodríguez-Navarro, D.; Lázaro-Galilea, J.L.; Bravo-Muñoz, I.; Gardel-Vicente, A.; Tsirigotis, G. Analysis and calibration of sources of electronic error in PSD sensor response. Sensors 2016, 16, 619. [CrossRef] [PubMed]

(C) 2016 by the authors; licensee MDPI, Basel, Switzerland. This article is an open access article distributed under the terms and conditions of the Creative Commons Attribution (CC-BY) license (http://creativecommons.org/licenses/by/4.0/). 